ORIGINAL RESEARCH

\title{
Coronary Risk Assessment by Point-Based vs. Equation-Based Framingham Models: Significant Implications for Clinical Care
}

\author{
William J. Gordon, BA ${ }^{7,2}$, Jesse M. Polansky, MD, MPH ${ }^{3}$, W. John Boscardin, PhD ${ }^{4,5}$, \\ Kathy Z. Fung, $M S^{4,6}$, and Michael A. Steinman, $M D^{2,4,6}$
}

${ }^{1}$ Weill Cornell Medical College, New York, NY, USA; ${ }^{2}$ Medical Student Training in Aging Research Program, San Francisco, CA, USA; ${ }^{3}$ Centers
for Medicare and Medicaid Services, Baltimore, MD, USA; ${ }^{4}$ Division of Geriatrics, University of California, San Francisco, CA, USA; ${ }^{5}$ Department
of Epidemiology and Biostatistics, University of California, San Francisco, CA, USA; ${ }^{6}$ San Francisco VA Medical Center, San Francisco, CA, USA.

BACKGROUND: US cholesterol guidelines use original and simplified versions of the Framingham model to estimate future coronary risk and thereby classify patients into risk groups with different treatment strategies. We sought to compare risk estimates and risk group classification generated by the original, complex Framingham model and the simplified, pointbased version.

METHODS: We assessed 2,543 subjects age 20-79 from the 2001-2006 National Health and Nutrition Examination Surveys (NHANES) for whom Adult Treatment Panel III (ATP-III) guidelines recommend formal risk stratification. For each subject, we calculated the 10year risk of major coronary events using the original and point-based Framingham models, and then compared differences in these risk estimates and whether these differences would place subjects into different ATP-III risk groups $(<10 \%$ risk, $10-20 \%$ risk, or $>20 \%$ risk). Using standard procedures, all analyses were adjusted for survey weights, clustering, and stratification to make our results nationally representative.

RESULTS: Among 39 million eligible adults, the original Framingham model categorized $71 \%$ of subjects as having "moderate" risk $(<10 \%$ risk of a major coronary event in the next 10 years), 22\% as having "moderately high" (10$20 \%$ ) risk, and $7 \%$ as having "high" (>20\%) risk. Estimates of coronary risk by the original and point-based models often differed substantially. The point-based system classified $15 \%$ of adults (5.7 million) into different risk groups than the original model, with 10\% (3.9 million) misclassified into higher risk groups and 5\% (1.8 million) into lower risk groups, for a net impact of classifying 2.1

This research was presented at the annual meetings of the American Geriatrics Society (Chicago, April 30, 2009) and the Society of General Internal Medicine (Miami, May 14, 2009) and at the Bay Area Clinical Research Symposium (San Francisco, October 3, 2008)

Dr. Polansky's contribution to this article is made in his personal capacity, not as a Centers for Medicare Medicaid Services employee. The opinions (or conclusions) expressed are those of Dr. Polansky and not necessarily of CMS or the US Department of Health and Human Services.

Electronic supplementary material The online version of this article (doi:10.1007/s11606-010-1454-2) contains supplementary material, which is available to authorized users.

Received September 23, 2009

Revised April 1, 2010

Accepted June 8, 2010

Published online September 8, 2010 million adults into higher risk groups. These risk group misclassifications would impact guideline-recommended drug treatment strategies for 25-46\% of affected subjects. Patterns of misclassifications varied significantly by gender, age, and underlying CHD risk.

CONCLUSIONS: Compared to the original Framingham model, the point-based version misclassifies millions of Americans into risk groups for which guidelines recommend different treatment strategies.

KEY WORDS: risk assessment; cholesterol; heart diseases; practice guidelines as topic; prognosis.

J Gen Intern Med 25(11):1145-51

DOI: $10.1007 / \mathrm{s} 11606-010-1454-2$

(c) The Author(s) 2010. This article is published with open access at Springerlink.com

\section{INTRODUCTION}

National cholesterol guidelines use a "Framingham model" to calculate a person's 10-year risk of myocardial infarction or coronary death. Based on this risk, patients are categorized into different risk groups, which are used to guide treatment decisions. ${ }^{1}$ The Framingham model is a complex mathematical equation. To enable use in settings without calculators or computers, the formula for calculating risk was simplified into a point-based or "score sheet" system. ${ }^{2}$

Both the original and point-based versions of the Framingham model are endorsed by the National Cholesterol Education Project's Adult Treatment Panel III (ATP III) guidelines. ${ }^{1,3}$ However, it is possible that the simplified point-based system may lead to less accurate risk estimates and potentially different treatment recommendations. The original system derives risk estimates using Cox regression models based on patient age, total and HDL cholesterol, systolic blood pressure, treatment for hypertension, and smoking status. For example, based on the original Framingham model equation, a 45-year-old male smoker with total cholesterol $170 \mathrm{mg} / \mathrm{dl}$, HDL $38 \mathrm{mg} / \mathrm{dl}$, and systolic blood pressure of $125 \mathrm{mmHg}$ not on antihypertensive treatment would be calculated to have a $7 \%$ risk of myocardial infarction or death over 10 years. In contrast, the point-based system assigns each risk factor level an integer number. These risk factor values are summed to derive a score, and then the risk for that score is determined from a look-up table. For example, this same patient above would get 3 points for being age 
45-49 years, 5 points for smoking, and so forth for a total score of 13 points, which corresponds to a $12 \%$ risk of a major coronary event over the next 10 years.

The proliferation of computers and personal digital assistants (PDAs) in clinical medicine enables easy implementation of the original, mathematically complex Framingham model at the point of care. However, the point-based system has remained in widespread use in both clinical practice and research, including widespread application in computerized risk prediction tools. ${ }^{4-8}$ Given that approximately 36 million persons in the US are eligible for lipidlowering therapy, differences in classification could result in millions of persons receiving different lipid-lowering therapy depending on which model is used. ${ }^{9,10}$ In this study, we used nationally representative data to compare differences in predicted risk between the original and point-based Framingham calculations and to determine the degree to which the point-based system stratifies patients into different risk groups.

\section{METHODS}

Data were obtained from the 2001-2006 waves of the National Health and Nutrition Examination Survey (NHANES). Respondents were selected and their data weighted to be representative of the non-institutionalized US population. ${ }^{11}$

Under ATP III guidelines, patients with known coronary heart disease (CHD) or risk equivalents are considered high-risk ( $>20 \%$ 10 -year risk). Patients with 0-1 risk factors are considered to be low risk. Patients with two or more risk factors but no known CHD or risk equivalents are placed in an indeterminate risk group. The Framingham risk model is used on patients in this indeterminate group to assess whether they are at "moderate" $(<10 \%)$, "moderately high" (10-20\%), or "high" ( $>20 \%)$ risk of myocardial infarction or coronary death in the next 10 years (Fig. 1). ${ }^{1}$

Our analyses focused on adults age 20-79 in this indeterminate group (the Framingham models are not adapted to people over age 79 years). We excluded the $11 \%$ of patients in this group who reported taking lipid-lowering therapy, since the Framingham models and risk stratification algorithms are not designed to predict risk in this population.

We used self-report data from NHANES to exclude subjects with CHD or risk equivalents, including myocardial infarction, angina pectoris, diabetes mellitus, or stroke. Next, we summed the number of risk factors for each subject including current cigarette use, hypertension (defined by self-report or documented blood pressure $\geq 140 / \geq 90 \mathrm{mmHg}$ ), low HDL ( $<40 \mathrm{mg} / \mathrm{dl}$ ), family history of CHD (history of heart attack or angina before age 50 years in close biological relatives), and age (male $>45$ years old, female $>55$ years old). Subjects with $\mathrm{HDL}>60 \mathrm{mg} / \mathrm{dl}$ had one point subtracted from their risk factor sum. Subjects with two or more risk factors formed our analytic cohort.

We used the multiple imputation by chained equations procedure for the 2005-2006 wave of NHANES to account for the approximately $11 \%$ of eligible subjects who had incomplete data, mostly due to absent blood pressures and laboratory values for total and HDL cholesterol. ${ }^{12,13}$ Our multiple imputation analysis results were very similar to those obtained analyzing only subjects with complete data. We thus conducted our main analyses for all three waves on subjects with complete data.

\section{Analyses}

As the Framingham models are gender specific, we split our cohort into male and female groups. Using formulas published on the Framingham Heart Study website, for each subject we calculated the predicted 10-year risk of hard CHD events using the original and point-based models. ${ }^{14}$ Next, we determined which risk group each person in our cohort would be placed in under the original model and calculated the number of people reclassified into a higher or lower risk group by the pointbased system. We term this shift between risk groups "misclassification," insofar as such patients are misclassified relative to the original Framingham model. We evaluated differences in risk classification using kappa statistics and compared the estimated probabilities of misclassification into lower or higher ATP III risk groups using multinomial models.

Finally, among patients misclassified by the point-based system, we determined whether this misclassification would impact guideline-based drug treatment recommendations. For this analysis, we used LDL levels, which were collected on NHANES participants examined in the morning (1,079 of our 2,543 subjects). Among misclassified subjects, we determined if LDL levels were above the threshold for starting drug therapy given their risk group determined by one model, but below the threshold for starting drug therapy given the risk group determined by the other model.

All analyses were conducted using Stata 10.1 (StataCorp, College Station, TX) and were adjusted for subject weights and clustering effects using standard methods recommended by NHANES to make our results nationally representative.

\section{RESULTS}

Among 11,967 subjects aged 20-79 years, 1,898 had known CHD or risk equivalents, 7,080 had 0 or 1 CHD risk factors, and 446 were taking a lipid-lowering drug. The remaining 2,543 subjects, representing 39 million adults, formed our analytic cohort (Fig. 1). About one third of our analytic cohort was female, with a median age of 48 years, and three-quarters were white (Table 1). Half were cigarette smokers, 56\% had hypertension, 30\% had a family history of CHD, 46\% had HDL cholesterol $<40 \mathrm{mg} / \mathrm{dl}$, and slightly under half had LDL cholesterol levels of $130 \mathrm{mg} / \mathrm{dl}$ or greater. As calculated by the original model, $71 \%$ had a "moderate" risk of a major coronary event in the next 10 years (including $48 \%$ with $<5 \%$ risk and 23\% with 5-9\% risk), 22\% were at "moderately high" risk, and $7 \%$ were at "high" risk (data not shown in Table).

Figure 2 shows the ATP III risk groups that patients would be assigned to had their risk been calculated by the original model vs. the point-based model. Compared with the original model, the point-based system misclassified $15 \%$ of subjects (95\% CI, 13\%-16\%) into different ATP III risk groups, corresponding to 5.7 million people. Kappa for agreement in risk group stratification was 0.69. Misclassification disproportionately shifted patients into higher risk groups $(\mathrm{P}<0.001)$, with 10\% (95\% CI 9\%-12\%; 3.9 million people) misclassified into higher risk groups and 5\% (95\% CI, 4\%-6\%; 1.8 million people) into lower risk groups. The majority of upward classification originated among patients in the lowest risk group. The largest source of downward classification was 


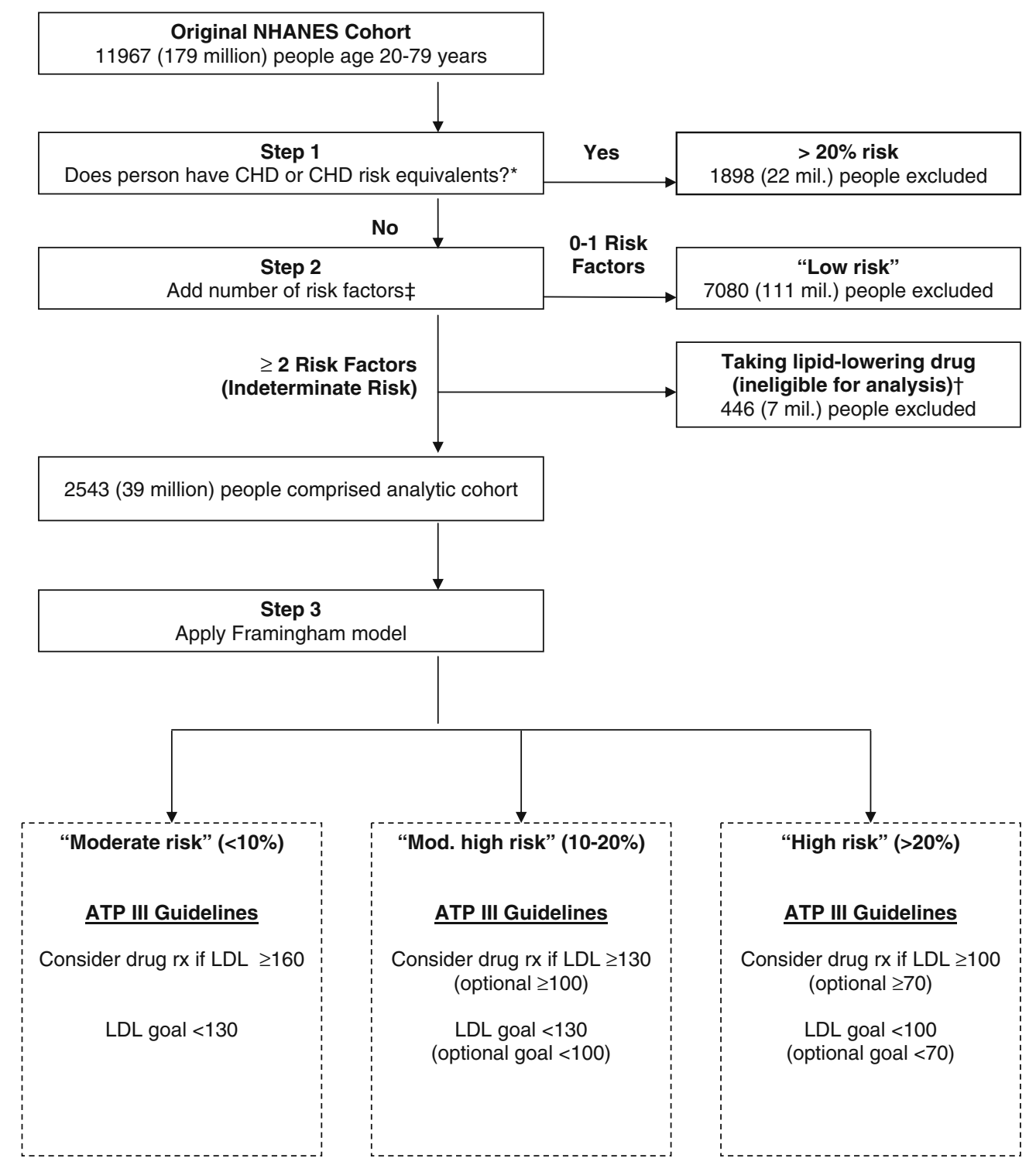

Figure 1. Creation of analytic cohort and LDL treatment guidelines for patients undergoing Framingham-based risk stratification. ${ }^{*} \mathrm{CHD}$ risk equivalents included self-report of myocardial infarction, angina pectoris, diabetes mellitus, and stroke. †Persons taking lipid-lowering drugs ineligible for analysis. ₹CHD risk factors included: cigarette smoking, hypertension, $\mathrm{HDL}<\mathbf{4 0} \mathrm{mg} / \mathrm{dl}$, family history of premature CHD, and age (>45 years for men, $>55$ years for women). Persons with HDL $>60 \mathrm{mg} / \mathrm{dl}$ had 1 point subtracted from their risk factor sum score.

among subjects placed in the "high risk" group by the original Framingham model, of whom $45 \%$ ( 1.2 million of 2.7 million) were misclassified as "moderately high risk" or "moderate risk" under the point-based system.

Patterns of misclassification varied significantly by gender and age. Overall, $17 \%$ of men (95\% CI, 15\%-19\%; 4.3 million) and $11 \%$ of women (95\% CI, 9\%-14\%; 1.4 million) were misclassified $(\mathrm{P}=0.003$ for difference between genders). Among those misclassified, $64 \%$ of men $195 \%$ CI, $58 \%-$ $70 \%)$ and $80 \%$ of women (95\% CI, $72 \%-87 \%)$ were shifted into higher risk groups by the point-based system. Results also varied by age groups. Misclassification affected $7 \%$ of people aged 20-44 years (95\% CI, 5\%-9\%; 1.0 million), $17 \%$ aged 45-64 years (95\% CI, 15\%-20\%; 3.2 million), and $27 \%$ aged 65-79 years (95\% CI, 23\%-30\%, 1.5 million; $\mathrm{P}<0.001$ for difference between age groups). This variation in misclassification patterns may in part be attributable to underlying differences in CHD risks between age and sex groups.

Next, we compared point estimates of risk generated by the original and point-based models. On average, the point-based system generated higher risk estimates than the original model by a mean of $0.6 \%$ (95\% CI, $0.5 \%-0.8 \%$; SD $3.3 \%$ ) in men and $0.4 \%(95 \% \mathrm{CI}, 0.2 \%-0.6 \%$, SD $2.5 \%)$ in women (Table 2 and e-Appendix). Differences between the models were often substantial for individual patients, and the magnitude of differences grew as risk increased. For example, the median absolute risk difference for men at "moderate" risk (predicted by the original model) was $1.0 \%$ [interquartile range (IQR) $0.5 \%-1.8 \%$ ), increasing to a mean absolute risk difference of $3.6 \%$ (IQR 1.8\%-6.4\%) for those in the "high risk" group. Differences of more than $5 \%$ between the original and point-based models were common at higher levels of risk, occurring in $26 \%$ of subjects (95\% CI, 23\%-30\%) who had 
Table 1. Characteristics of Sample

\begin{tabular}{ll}
\hline \hline Characteristic & $\%$ of sample* \\
\hline Age, median (interquartile range) & $48(38-59)$ \\
Female & $34 \%$ \\
Race & \\
White & $78 \%$ \\
Black & $9 \%$ \\
Asian/PI & $9 \%$ \\
Other & $4 \%$ \\
Current cigarette smoker & $50 \%$ \\
Hypertension & $56 \%$ \\
Family history of CHD & $30 \%$ \\
HDL <40 mg/dl & $46 \%$ \\
LDL cholesterol (mg/dl) & \\
$<100$ & $22 \%$ \\
$100-129$ & $33 \%$ \\
$130-159$ & $30 \%$ \\
$\geq 160$ & $15 \%$ \\
\hline
\end{tabular}

$* N=2,543$ subjects. LDL cholesterol was assessed in 1,079 subjects whose laboratory tests were drawn in the morning

risk scores above $10 \%$ as calculated by the original model (data not shown in Table).

Figure 3 shows results from the perspective of point-based scores. For each level of point-based risk, the box-andwhisker format shows the distribution of risk estimates calculated by the original model. For example, consider women with a point-based risk estimate of $8 \%$. The upper border of the box corresponds to the upper 25th percentile of risk estimates calculated by the original model. Since the upper border of the box lies at 10\% risk, approximately onequarter of women with point-based scores of $8 \%$ had original model scores of $10 \%$ or above. As such, one-quarter of women with a point-based score of $8 \%$ would be reclassified from the "moderate risk" category ( $<10 \%$ risk) to the "moderately high risk" category (10-20\% risk) had the original model been used instead. Overall, for women and men with point-based scores above 6\%, reclassification into different ATP III risk strata was common. In contrast, very few subjects with point-based scores of $6 \%$ or less would have been classified into the different ATP III risk groups had the original model been used instead of the point-based version.

Finally, we evaluated the potential impact of risk group misclassification on guideline-recommended treatment decisions. Under standard cutpoints of the original ATP III guidelines, $25 \%$ of subjects (95\% CI, $17 \%$ to $36 \%$ ) misclassified by the point-based model would have had different drug treatment strategies recommended as a result of misclassification, with $18 \%$ (95\% CI, 11\%-26\%) recommended for more intensive treatment and 7\% (95\% CI, 4\%-12\%) recommended for less intensive treatment $(\mathrm{P}=0.01$ for direction of treatment effects). Using more aggressive optional targets published in a 2004 update to ATP III, $46 \%$ of subjects (95\% CI, 37\% to 56\%) misclassified by the point-based model would have drug treatment recommendations changed as a result of misclassification, including 39\% (95\% CI, 31\%-48\%) being recommended for more intensive therapy and 7\% (95\% CI, 4\%-13\%) for less intensive therapy $(\mathrm{P}<0.001$ for direction of treatment effects).

\section{DISCUSSION}

In this nationally representative study, the original and pointbased Framingham models produced clinically meaningful dif- ferences in estimated CHD risk for many individuals and stratified substantial numbers of patients into different risk groups established by ATP III guidelines. Overall, the point-based system classified 15\% of eligible Americans (5.7 million people) into different risk groups than the original Framingham model. Misclassification predominantly shifted patients into higher risk groups, with $10 \%$ of adults (3.9 million) misclassified into higher risk groups and 5\% (1.8 million) into lower risk groups, and had the potential to impact drug treatment recommendations in 25$46 \%$ of affected subjects not currently taking lipid-lowering therapy. Because our analyses excluded study subjects with incomplete data, patients on lipid-lowering therapy, and patients who in clinical practice may receive Framingham risk prediction outside formal guideline criteria, our results underestimate the number of Americans potentially affected by differences in the point-based and original Framingham models. ${ }^{6}$

These discrepancies comprise one of the ongoing challenges in a history of impressive advances in cardiovascular risk assessment. Beginning with a sum-of-risk-factors approach in the first report of the National Cholesterol Education Program, successive advances in modeling have improved clinicians' ability to predict—and thereby better prevent—cardiovascular events. ${ }^{15-18}$ As predictive models became more complex and impractical to calculate by hand, point-based versions became necessary to facilitate their regular use. This need may persist in settings where computer-based risk calculators are not readily available at the point of care, as it may be preferable to have an imperfect system of risk prediction than none at all. However, as the availability of desktop- and handheld-based computers has become routine in clinical practice, there is limited need for predictive models that can be calculated using pen and paper.

Nonetheless, the point-based system remains in widespread use, including in risk calculators on websites and personal digital assistants, and such tools are often not transparent in noting which model they use. Thus, the misclassifications of risk that we observed are likely common in clinical practice, and may have substantial clinical and policy implications. Of particular note, over two-thirds of misclassifications moved patients into higher risk groups. Because guidelines recommend more aggressive treatment strategies for patients in higher risk groups, this misclassification may drive increases in the use of lipid-lowering medications. This may have some benefits by reducing cardiovascular event rates, although at the risk of increasing adverse drug reactions, patients' medication

\begin{tabular}{|c|c|c|c|c|c|}
\hline \multirow{3}{*}{\multicolumn{2}{|c|}{ Moderate risk }} & & & & \\
\hline & & Moderate risk & $\begin{array}{l}\text { Moderately } \\
\text { high risk }\end{array}$ & High risk & \multirow[b]{2}{*}{$27.4 \mathrm{~m}(71 \%)$} \\
\hline & & $24.2 \mathrm{~m}(63 \%)$ & $3.2 \mathrm{~m}(8 \%)$ & $<0.1 \mathrm{~m}(<1 \%)$ & \\
\hline \multirow[t]{3}{*}{$\begin{array}{l}\text { Original } \\
\text { model }\end{array}$} & $\begin{array}{r}\text { Moderately } \\
\text { high risk }\end{array}$ & $0.6 \mathrm{~m}(2 \%)$ & $7.3 \mathrm{~m}(19 \%)$ & $0.7 \mathrm{~m}(2 \%)$ & $8.6 \mathrm{~m}(22 \%)$ \\
\hline & High risk & $<0.1 \mathrm{~m}(<1 \%)$ & $1.2 \mathrm{~m}(3 \%)$ & $1.5 \mathrm{~m}(4 \%)$ & $2.7 \mathrm{~m}(7 \%)$ \\
\hline & & $24.8 \mathrm{~m}(64 \%)$ & $11.6 \mathrm{~m}(30 \%)$ & $2.2 \mathrm{~m}(6 \%)$ & $38.7 \mathrm{~m}(100 \%$ \\
\hline
\end{tabular}

Figure 2. Classification of Subjects into Risk Groups by the PointBased and Original Model. *Cells to the right of the diagonal represent the point-based system estimating a higher risk than the original model. Cells to the left of the diagonal represent the pointbased system estimating a lower risk than the original model. Overall, 2,543 subjects contributed data toward this table 
Table 2. Differences in Estimated Risk by Original Framingham Model and Point-Based System, by Level of Risk

\begin{tabular}{|c|c|c|c|c|}
\hline & \multicolumn{4}{|l|}{ Men } \\
\hline & Overall & "Moderate risk" & "Moderately high risk" & "High risk" \\
\hline $\begin{array}{l}\text { Risk difference between models - } \\
\text { mean (SD), [range] }\end{array}$ & $\begin{array}{l}0.6 \%(3.3 \%) \\
{[-29.4 \% \text { to } 14.5 \%]}\end{array}$ & $\begin{array}{l}0.9 \%(1.5 \%) \\
{[-4.4 \% \text { to } 10.3 \%]}\end{array}$ & $\begin{array}{l}0.9 \%(4.1 \%) \\
{[-7.9 \% \text { to } 14.5 \%]}\end{array}$ & $\begin{array}{l}-1.9 \%(7.7 \%) \\
{[-29.4 \% \text { to } 9.7 \%]}\end{array}$ \\
\hline \multirow[t]{3}{*}{$\begin{array}{l}\text { Absolute risk difference between } \\
\text { models - median (IQR) }\end{array}$} & $\begin{array}{l}1.4 \% \\
(0.6 \% \text { to } 2.9 \%)\end{array}$ & $\begin{array}{l}1.0 \% \\
(0.5 \% \text { to } 1.8 \%)\end{array}$ & $\begin{array}{l}2.3 \% \\
(1.1 \% \text { to } 4.7 \%)\end{array}$ & $\begin{array}{l}3.6 \% \\
(1.8 \% \text { to } 6.4 \%)\end{array}$ \\
\hline & \multicolumn{4}{|l|}{ Women } \\
\hline & Overall & "Moderate risk" & "Moderately high risk" & "High risk" \\
\hline $\begin{array}{l}\text { Risk difference between models - } \\
\text { mean (SD), [range] }\end{array}$ & $\begin{array}{l}0.4 \%(2.5 \%) \\
{[-20.2 \% \text { to } 12.1 \%]}\end{array}$ & $\begin{array}{l}0.4 \%(1.7 \%) \\
{[-4.6 \% \text { to } 12.1 \%]}\end{array}$ & $\begin{array}{l}1.6 \%(5.3 \%) \\
{[-7.6 \% \text { to } 11.6 \%]}\end{array}$ & $\dagger$ \\
\hline $\begin{array}{l}\text { Absolute risk difference between } \\
\text { models - median (IQR) }\end{array}$ & $\begin{array}{l}0.6 \% \\
(0.2 \% \text { to } 1.7 \%)\end{array}$ & $\begin{array}{l}0.5 \% \\
(0.2 \% \text { to } 1.4 \%)\end{array}$ & $\begin{array}{l}3.1 \% \\
(1.6 \% \text { to } 5.3 \%)\end{array}$ & $\dagger$ \\
\hline
\end{tabular}

*Risks bands shown at top of the table were calculated using the original model. Positive numbers in the first row (means) correspond to the point-based system predicting higher risk than the original model; negative numbers correspond to the opposite

$\dagger$ Estimates of differences between the two models are unreliable for women at $>20 \%$ risk due to low numbers of subjects in this risk group in the NHANES sample

burden, and clinician time and resources. ${ }^{19}$ In addition, there is debate over the utility of expanding drug therapy beyond NCEP guidelines. ${ }^{3,20-30}$

Also concerning is potential undertreatment for the 1.8 million people whom the point-based system misclassifies into lower risk groups, particularly the nearly $50 \%$ of people (1.2 million) at high coronary risk whom the point-based system triages into lower risk categories. Failure to define and pursue aggressive LDL goals in such patients may compound the widespread undertreatment of persons at high cardiovascular risk. ${ }^{10}$

Unfortunately, there does not appear to be a simple "fix" to correct the misclassification that occurred under the point- based system. Patterns of misclassification were complex, varying by underlying CHD risk, sex, and age. In addition, the population-level implications of misclassification also vary among age and sex groups. For example, a substantial majority $(80 \%)$ of the 1.4 million misclassified women were misclassified into higher risk groups, largely reflecting the fact that most women had calculated risk of under $10 \%$ by the original model, so their only available direction for risk group misclassification was into a higher risk group. In contrast, while misclassification also predominantly placed men into higher risk groups, a substantial minority (36\%) were shifted into lower risk groups, leaving them susceptible to undertreatment. This was particularly notable for the 2.5
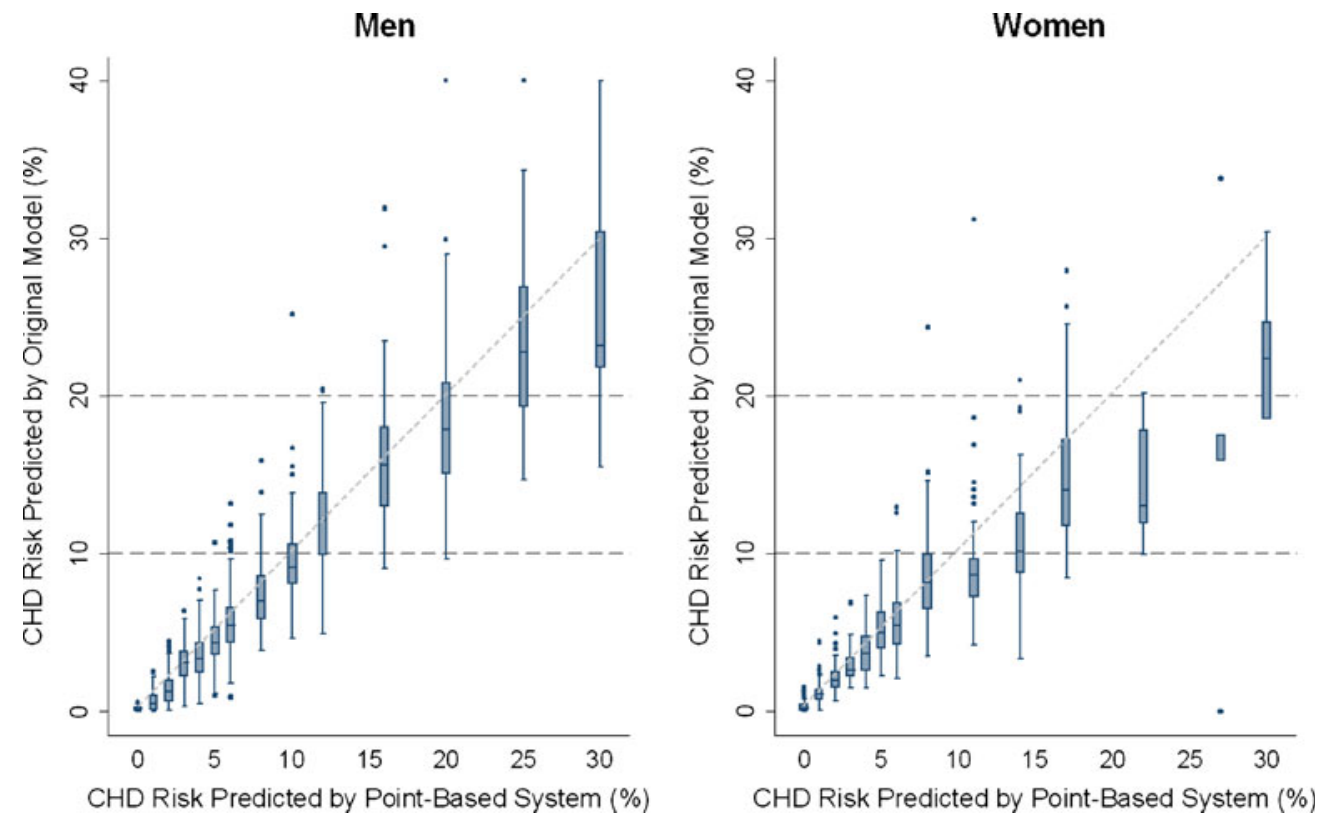

Figure 3. Classification of Subjects into Risk Groups by the Point-Based and Original Model. *Cells to the right of the diagonal represent the point-based system estimating a higher risk than the original model. Cells to the left of the diagonal represent the point-based system estimating a lower risk than the original model. Overall, 2,543 subjects contributed data toward this table 
million men at high risk of future coronary events, almost half of whom were misclassified by the point-based model into a lower risk group.

ATP III guidelines acknowledge that the original model gives more precise estimates of risk than the point-based one, but note that use of the point-based system "provide[s] a result that is accurate for clinical purposes." 31 While the differences in predicted risk between the models are small for the majority of patients, there are substantial numbers of patients for whom the two models produce clinically meaningful differences in predicted risk. National guidelines would benefit from acknowledging the calculated discrepancies between the two models and educating and guiding clinicians about preferred methods of risk stratification. More importantly, current guidelines should strongly consider endorsing the original model as the preferred method of risk calculation and as the sole appropriate option for computer or PDAbased risk calculators. In addition, patients and clinicians who made treatment decisions based on the point-based system should consider recalculating risk based on the original Framingham model and where appropriate adjust treatment plans accordingly.

Our results should be interpreted in the context of known limitations of the original Framingham model and previous evaluations of the NCEP risk stratification algorithm. ${ }^{6,7,32}$ The original Framingham model has only moderate ability to distinguish between persons who will or will not have future coronary events (with ROC curves from validation studies mostly in the range of 0.65 to 0.75$).{ }^{33-36}$ This model was also developed in a mostly white, middle-class population, and validation studies have revealed that it overestimates CHD risk in a number of other populations. ${ }^{33-36}$ Other research suggests that Framingham-based risk assessment should be expanded to patients with 0 or 1 risk factors. ${ }^{6,7}$ In addition, national guidelines from other countries use versions of the Framingham model in different, often more conservative ways to guide lipid management. ${ }^{37}$ In the US, it is well-documented that many patients - particularly those at high coronary riskhave LDL levels above current guideline recommendations. ${ }^{10}$ Thus, our findings should be interpreted as one piece of a larger challenge of appropriately identifying individuals' coronary risk profiles and increasing adherence to treatment strategies optimally tailored to those individuals' risk.

The next generation of cholesterol guidelines (ATP IV) is expected to be released in the near future, and it is likely that these new guidelines will predict risk using a new model of global cardiovascular risk prediction that incorporates a broader range of cardiovascular outcomes. ${ }^{35}$ Nonetheless, score-sheet versions of this model have already been developed and if applied to guidelines may result in problems similar to those that we observed. ${ }^{35}$ Thus, when simplifying future models of cardiovascular or other forms of risk, it will be essential to account for the practical effects of simplification on algorithm-based management decisions and to disseminate these analyses in peerreviewed publications to maximize transparency. ${ }^{38}$

There are several limitations to our study. Our estimates of how many subjects would be recommended for changes in lipidlowering therapy based on misclassification are approximate due to limited sample sizes, absence of data on potential lifestyle interventions, and potential inaccuracies in self-reported use of lipid-lowering medications. In addition, we did not evaluate users of lipid-lowering drugs, so we do not know what the impact of using different Framingham models would have been prior to their initiating drug treatment. Finally, our study did not have access to actual cardiovascular outcomes, and so we are unable to determine the accuracy of these models for predicting cardiovascular events. Nonetheless, the original Framingham model is the de facto gold standard for ATP III-based risk prediction, and mathematically is it very unlikely that a pointbased system derived from the original model would be more accurate than the original model itself.

In summary, the point-based Framingham risk prediction tool misclassifies millions of Americans into different ATP III risk groups compared with the original Framingham model, with 25$46 \%$ of affected subjects experiencing potential impacts on drug treatment recommendations. Guidelines and their associated risk prediction tools should account for the clinically meaningful differences that can arise between original and point-based models and the impact that these differences can have on treatment decisions. This will support the goal of a clinically consistent, transparent, and standardized approach to cardiovascular risk assessment.

Acknowledgements: The authors thank Kenneth Covinsky, MD, MPH for his insights into the development of this research.

Funding: This work was supported by the Medical Student Training in Aging Research (MSTAR) program of the National Institute of Aging, the American Federation for Aging Research, and the Hartford Foundation (Mr. Gordon) and by a Career Development Transition Award 01-013 from the VA Health Services Research and Development Service and a Paul Beeson award K23AG030999 from the National Institutes of Health and the American Federation for Aging Research (Dr. Steinman). These funding sources had no role in the design and conduct of this study; collection, management, analysis, and interpretation of the data; or preparation, review, and approval of the manuscript. The opinions (and conclusions) expressed in this article are those of the authors and do not necessarily reflect the official views of the Centers for Medicare and Medicaid Services, the United States Department of Health and Human Services, or the Department of Veterans Affairs.

Disclosure: Dr. Polansky is lead plaintiff in United States of America ex rel. Polansky v. Pfizer, Inc., 04-cv-0704 (ERK) (E.D.N.Y.). This litigation alleges that Pfizer, which manufactures the lipidlowering agent Lipitor (atorvastatin), conducted an improper marketing campaign to encourage unnecessary use of Lipitor. One of the allegations in the plaintiff's complaint was that Pfizer promoted the use of the point-based version of the Framingham model in order to increase Lipitor sales, including through wide distribution of the paper-based point-based risk calculator, using the point-based calculation in its Lipitor website, and funding the ePocrates cholesterol management PDA software that employs the point-based Framingham system.

Ethical Approval: This research was approved by the institutional review boards of the San Francisco VA Medical Center and the University of California, San Francisco.

Data Access and Responsibility: Dr. Steinman had full access to all the data in the study and takes responsibility for the integrity of the data and the accuracy of the data analysis.

Open Access: This article is distributed under the terms of the Creative Commons Attribution Noncommercial License which permits any noncommercial use, distribution, and reproduction in any medium, provided the original author(s) and source are credited.

Corresponding Author: Michael A. Steinman, MD; San Francisco VA Medical Center, 4150 Clement St, Box 181G, San Francisco, CA 94121, USA (e-mail: mike.steinman@ucsf.edu). 


\section{REFERENCES}

1. Executive Summary of The Third Report of The National Cholesterol Education Program (NCEP) Expert Panel on Detection, Evaluation, And Treatment of High Blood Cholesterol In Adults (Adult Treatment Panel III). Jama. 2001;285:2486-97.

2. Sullivan LM, Massaro JM, D'Agostino RB Sr. Presentation of multivariate data for clinical use: The Framingham Study risk score functions. Stat Med. 2004;23:1631-60.

3. Grundy SM, Cleeman JI, Merz CN, et al. Implications of recent clinical trials for the National Cholesterol Education Program Adult Treatment Panel III guidelines. Circulation. 2004;110:227-39.

4. Sheridan S, Pignone M, Mulrow C. Framingham-based tools to calculate the global risk of coronary heart disease: a systematic review of tools for clinicians. J Gen Intern Med. 2003; 18: 1039-52.

5. Epocrates Rx STAT Cholesterol application on Palm Pilot portable digital assistant platform. Available at: www.epocrates.com. Accessed June 17 2010.

6. Persell SD, Lloyd-Jones DM, Baker DW. National Cholesterol Education Program risk assessment and potential for risk misclassification. Prev Med. 2006;43:368-71.

7. Ajani UA, Ford ES. Two approaches of coronary risk assessment-do they work? Am J Cardiol. 2006;97:765-7.

8. MayoClinic.com Heart Disease Risk Calculator. Available at: http:// www.mayoclinic.com/health/heart-disease-risk/HB00047. Accessed June 15, 2010

9. Fedder DO, Koro CE, L'Italien GJ. New National Cholesterol Education Program III guidelines for primary prevention lipid-lowering drug therapy: projected impact on the size, sex, and age distribution of the treatment-eligible population. Circulation. 2002;105:152-6.

10. Persell SD, Lloyd-Jones DM, Baker DW. Implications of changing national cholesterol education program goals for the treatment and control of hypercholesterolemia. J Gen Intern Med. 2006;2 1:171-6.

11. National Center for Health Statistics. The National Health and Nutrition Examination Survey (NHANES) Analytic and Reporting Guidelines (update version September 2006). Hyattsville, MD. Available at: http:// www.cdc.gov/nchs/nhanes/nhanes2003-2004/analytical guidelines. htm. Accessed June 15, 2010

12. Royston P. Multiple imputation of missing values. Stata Journal. 2004;4:227-241.

13. van Buuren S, Boshuizen HC, Knook DL. Multiple imputation of missing blood pressure covariates in survival analysis. Statistics in Medicine. 1999; 18:681-694.

14. National Heart Lung and Blood Institute. Framingham Heart Study: Hard Coronary Heart Disease: 10-year risk. Available at: http://www. framinghamheartstudy.org/risk/hrdcoronary.html. Accessed June 15, 2010

15. Wilson PW, D'Agostino RB, Levy D, Belanger AM, Silbershatz $\mathbf{H}$, Kannel WB. Prediction of coronary heart disease using risk factor categories. Circulation. 1998;97:1837-47.

16. Wilson PWF, Pencina MJ, Jacques $\mathbf{P}$, Selhub J, D'Agostino R Sr, O'Donnell CJ. C-reactive protein and reclassification of cardiovascular risk in the Framingham Heart Study. Circ Cardiovasc Qual Outcomes. 2008; 1:92-97.

17. Janes H, Pepe MS, Gu W. Assessing the value of risk predictions by using risk stratification tables. Ann Intern Med. 2008;149:751-60.

18. Cook NR, Ridker PM. Advances in measuring the effect of individual predictors of cardiovascular risk: the role of reclassification measures. Ann Intern Med. 2009;150:795-802.
19. Hippisley-Cox J, Coupland C. Unintended effects of statins in men and women in England and Wales: population based cohort study using the QResearch database. BMJ;340:c2197.

20. Law MR, Wald NJ, Thompson SG. By how much and how quickly does reduction in serum cholesterol concentration lower risk of ischaemic heart disease? BMJ. 1994;308:367-72.

21. Law MR, Wald NJ, Rudnicka AR. Quantifying effect of statins on low density lipoprotein cholesterol, ischaemic heart disease, and stroke: systematic review and meta-analysis. BMJ. 2003;326:1423.

22. Hayward RA, Hofer TP, Vijan S. Narrative review: lack of evidence for recommended low-density lipoprotein treatment targets: a solvable problem. Ann Intern Med. 2006;145:520-30.

23. Tinetti ME, Bogardus ST Jr, Agostini JV. Potential pitfalls of diseasespecific guidelines for patients with multiple conditions. N Engl $J$ Med. 2004;351:2870-4.

24. Lenzer J. US consumer body calls for review of cholesterol guidelines. BMJ. 2004;329:759.

25. Pignone M, Earnshaw S, Tice JA, Pletcher MJ. Aspirin, statins, or both drugs for the primary prevention of coronary heart disease events in men: a cost-utility analysis. Ann Intern Med. 2006;144:326-36.

26. Ward S, Lloyd Jones M, Pandor A, et al. A systematic review and economic evaluation of statins for the prevention of coronary events. Health Technol Assess. 2007;11:1-160. iii-iv.

27. Reidenberg MM. Benefit/risk ratio of statins in primary prevention. Clin Pharmacol Ther. 2008;83:498-500.

28. Golomb BA, McGraw JJ, Evans MA, Dimsdale JE. Physician response to patient reports of adverse drug effects: implications for patienttargeted adverse effect surveillance. Drug Saf. 2007;30:669-75.

29. Criqui MH, Golomb BA. Low and lowered cholesterol and total mortality. J Am Coll Cardiol. 2004;44:1009-10.

30. Pletcher MJ, Lazar L, Bibbins-Domingo $\mathbf{K}$, et al. Comparing impact and cost-effectiveness of primary prevention strategies for lipid-lowering. Ann Intern Med. 2009; 150:243-54.

31. National Cholesterol Education Program. Third Report of the National Cholesterol Education Program (NCEP) Expert Panel on Detection, Evaluation, and Treatment of High Blood Cholesterol in Adults (Adult Treatment Panel III): Final Report. National Institutes of Health Publication No. 02-5215; 2002.

32. Fornasini M, Brotons C, Sellares J, et al. Consequences of using different methods to assess cardiovascular risk in primary care. Fam Pract. 2006;23:28-33.

33. D'Agostino RB Sr, Grundy S, Sullivan LM, Wilson P. Validation of the Framingham coronary heart disease prediction scores: results of a multiple ethnic groups investigation. JAMA. 2001;286:180-7.

34. Simmons RK, Sharp S, Boekholdt SM, et al. Evaluation of the Framingham risk score in the European Prospective Investigation of CancerNorfolk cohort: does adding glycated hemoglobin improve the prediction of coronary heart disease events? Arch Intern Med. 2008;168:1209-16.

35. D'Agostino RB Sr, Vasan RS, Pencina MJ, et al. General cardiovascular risk profile for use in primary care: the Framingham Heart Study. Circulation. 2008;117:743-53.

36. Brindle P, Emberson J, Lampe F, et al. Predictive accuracy of the Framingham coronary risk score in British men: prospective cohort study. BMJ. 2003;327:1267.

37. Manuel DG, Kwong $\mathbf{K}$, Tanuseputro $\mathbf{P}$, et al. Effectiveness and efficiency of different guidelines on statin treatment for preventing deaths from coronary heart disease: modelling study. BMJ. 2006;332:1419.

38. Woloshin S, Schwartz LM, Ellner A. Making sense of risk information on the web. BMJ. 2003;327:695-6. 